\title{
Genomic introgressions of immune Vitis rotundifola Michx into Russian grapevine germplasm revealed by RADseq genotyping
}

\author{
Potokina E.K. ${ }^{1,2 *}$, Grigoreva E.A. ${ }^{1,2,3}$, Karzhaev D.S. ${ }^{1}$, Ulianich P.S. ${ }^{4}$, Volkov V.A. ${ }^{1,2}$, \\ Vasylyk I.A. ${ }^{2}$, Volynkin V.A. ${ }^{2}$, Likhovskoi V.V. ${ }^{2}$ \\ ${ }^{1}$ Saint-Petersburg State Forestry University, St. Petersburg, Russia \\ ${ }^{2}$ All-Russian National Research Institute of Viticulture and Winemaking “Magarach” RAS, Yalta, Russia \\ ${ }^{3}$ ITMO University, St. Petersburg, Russia \\ ${ }^{4}$ All-Russia Research Institute for Agricultural Microbiology, St. Petersburg, Russia \\ *email: e.potokina@yahoo.com
}

Vitis vinifera L. is the most widely cultivated grape species in the world, and its plantations occupy about $94 \%$ of the area of the commercial vineyards. Traditionally, vineyards are protected against a disease by spraying, which has a harmful effect on the environment. Breeding of varieties resistant to the most common pathogens would allow switching to a more environmentally friendly way of cultivating grapes. Wild species of Vitis are generally disease resistant, but produce poor quality products. Breeding allows to combine disease resistance with grape quality traits using strategy of remote hybridizations.

The most promising source of resistance genes is the North American species $V$. rotundifolia Michx, which belongs to the subgenus Muscadiana Panch. Interspecific crosses of cultivated grapes with this species are extremely difficult, due to the different number of chromosomes. However, as a result of very few successful attempts, some interspecific hybrids with a stable karyotype were obtained in the beginning of the century. In 2011 their BC5 progenies have been involved into the breeding programs of "Magarach" Institute. Due to the lack of information about introgressed resistance loci and their localization on chromosomes, the breeding work is carried out "blindly", not allowing to manipulate by gene combinations to create genotypes resistant to the most dangerous diseases - downy and powdery mildew.

To solve the problem, 142 breeding lines from three half-sibling grape populations, carrying introgressions from $V$. rotundifolia, were "genotyped by sequencing" using RADseq method. In total, 309,762,340 high quality Illumina reads were generated. The sequencing data were aligned to the public available $V$. vinifera $12 \mathrm{X}$ genome assembly for SNP calling procedure. SNP calling was performed using Tassel V.5.2.40 and Stacks V. 2.53 bioinformatic software, yielding 45 thousands SNP per population. 12734 SNPs were shared between all three populations.

The genotyping data allowed to reveal a scheme of chromosome maps for each line, where introgressions of foreign material - fragments of the $V$. rotundifolia genome were located. Then, the introgressed regions of chromosomes that affect the variability of agrobiological and resistance traits will be decoded by sequencing to identify potential candidate genes.

Acknowledgements: The research was supported by the Russian Science Foundation (Project No. 20-16-00060). 